\title{
Computer simulation with DATASIM
}

\author{
DRAKE R. BRADLEY \\ Bates College, Lewiston, Maine
}

\begin{abstract}
A general purpose program is described for generating, analyzing, and graphing simulated data for experimental, multivariate, and contingency table designs. Applications include simulating actual or hypothetical research studies, conducting Monte Carlo investigations of the consequences of violating assumptions on statistical tests, generating randomized data sets for students to analyze and interpret as homework, and performing simulations demonstrating sampling theory. Data sets may be generated that have normal or nonnormal distributions, independent or correlated observations, and equal or unequal sample sizes. Data-analysis capabilities include oneway, two-way, and three-way ANOVA with any combination of independent- and correlated-groups factors, as well as simple-effects analyses, multiple pairwise comparisons, linear contrasts, and trend analyses.
\end{abstract}

The use of computer simulation for teaching and research is a relatively well-established practice in psychology. I first employed simulation in the mid-1970s, to teach principles of sampling theory in courses on statistics, experimental design, and research methods (Bradley, Hotchkiss, Dumais, \& Shea, 1976). The students in these courses would conduct simulations to assess the power of the $F$ test as a function of effect size, sample size, variability, and so forth. Somewhat later, I developed software for sampling contingency tables from userspecified populations. These programs were used for CAI demonstrations (Bradley \& Cutcomb, 1977), evaluation of the actual as opposed to the nominal Type I error rate of the chi-square statistic (Bradley, Bradley, McGrath, \& Cutcomb, 1979), and empirical determination of the power of the chi-square test (Bradley \& Seely, 1977). During this same period, I also developed software for generating independently randomized data sets for students to use in doing homework (Bradley, 1978; Bradley et al., 1976). While the data-generating algorithms were somewhat primitive, the program succeeded in providing students with simulated data sets based on actual studies, and in giving them feedback on the accuracy of their solutions.

It soon became apparent that one program could incorporate all of the capabilities described above. Of course, the data-analysis and graphics capabilities of the software would have to be fairly extensive, because simulations involving a wide variety of research designs and dataanalysis methods should be supported. The software package written to fulfill these requirements is called DATASIM (Bradley, 1988), for data simulator. The original mainframe version of this software (Bradley, 1984a, 1984b) has been supplanted by a more powerful and flexible version designed for the IBM and IBM-

Correspondence may be sent to Drake R. Bradley, Department of Psychology, Bates College, Lewiston, ME 04240. compatible PC (Bradley, 1988, in press). The latter version of DATASIM is the focus of this paper; versions for Macintosh and Amiga personal computers are in development.

DATASIM can generate simulated data for one-group, two-group, one-way, two-way, and three-way experimental designs; for correlational and multivariate designs; and for one- and two-way table (crossbreak) designs. DATASIM is also a reasonably comprehensive univariate statistical package, with extensive analysis of variance and analytical testing routines. In addition to the usual battery of statistical procedures (one- and two-sample tests, multiple correlation and regression, part and partial correlation, one- and two-way classification chi-square, etc.), DATASIM conveniently analyzes the data for one-way, two-way, and three-way experimental designs having any combination of independent- and correlated-groups factors. This includes simple-effects, simple-simple-effects, and simple-interaction tests, as well as trend analyses, multiple pairwise comparisons, and user-specified linear contrasts at the main effect, simple-effect, and simplesimple-effect levels. Pooled error terms are automatically selected for those tests and comparisons requiring them, and unweighted means analyses are conducted for factorial designs having unequal $n$ on one or more of the independent-groups factors. Consequently, although DATASIM was designed primarily for generating simulated data sets, researchers will find these data analysis capabilites very useful for analyzing their own raw data.

In sum, there are five major applications of DATASIM: (1) the simulation of hypothetical or actual research studies, (2) the Monte Carlo investigation of the Type I error rate or power of statistical tests, (3) the generation and analysis of simulated data for homework assignments, (4) the demonstration of sampling theory with laboratory assignments, and (5) the conducting of CAI demonstrations in the classroom. The remaining sections of this paper describe the first three applications in detail. For a discussion of applications involving laboratory as- 
signments and CAI demonstrations, see Bradley (1988, pp. 18, 94).

\section{Simulating Research Studies}

DATASIM allows one to easily generate, display, analyze, and graph simulated data for hypothetical or actual research studies. To generate simulated data, one must first initialize the simulation. This is done by entering commands that define the research design and any population parameters needed to control the simulation. Other commands specify sample sizes, distribution shapes, labels for conditions or variables, upper and lower limits for data values, the number of decimal places to retain in decimal data, and so forth. Although the command that specifies the design must be entered first, other initialization commands may be entered in any convenient order. Note that initializing a simulation allows DATASIM to generate simulated data with the properties desired, such as central tendency, variability, intercorrelation, precision, and distribution shape.

Once the simulation has been initialized, one can generate a simulated data set and analyze and graph the data. The value of the seed used to initialize the random number generator is displayed along with the simulated data, and this value may be used to regenerate the data at a later time. Also, once a simulation has been initialized, the design, labels, parameter values, and other information may be saved in a file so that the simulation can be reinitialized later.

For any given initialization, DATASIM can perform repetitive simulation; that is, it can generate a simulated data set, conduct one or more statistical analyses, plot the results, and then repeat this process over and over again. The output from the statistical analyses (e.g., means, $F$ tests, $t$ tests, etc.) can be saved in a buffer or file, subsequently trimmed of all text, and then reloaded as data into the DATASIM data buffer. The sampling distributions of the statistics thus loaded (as data) can then be displayed and analyzed. As shown in the next section, this capability is very useful for conducting sampling experiments that involve Monte Carlo methods.

As an illustration, consider a hypothetical study described in the DATASIM user manual (Bradley, 1988, p. 54). In this study, a researcher is interested in investigating the effectiveness of a new diet on reducing weight. It is suspected that the previous diet history of the individual, as either a frequent or infrequent dieter, may be a factor in the success of the dieting program. Therefore, $n=10$ frequent and $n=10$ infrequent dieters are solicited to participate in the study. Their weights are obtained before they start the diet, and after 1,2, and 3 weeks on it. This results in a $2 \times 4$ factorial design with independent groups on factor $\mathrm{A}$ (diet history), repeated measures on factor $B$ (weeks on diet), and $n=10 \mathrm{ob}$ servations per cell.

To generate simulated data we need to define the population means and standard deviations for each cell of the design, as well as the degree of intercorrelation between the repeated measurements taken over the 4 weeks. The population means will determine which effects (A, B, and $\mathrm{AB}$ ) are present, and which are not. Suppose that the cell means, $\mu_{\mathrm{ij}}$, are specified as follows:

$\begin{array}{lcccc} & \text { Before } & 1 \text { Week } & 2 \text { Weeks } & 3 \text { Weeks } \\ \text { Frequent } & \mu_{11}=145 & \mu_{12}=143 & \mu_{13}=141 & \mu_{14}=140 \\ \text { Infrequent } & \mu_{21}=159 & \mu_{22}=151 & \mu_{23}=147 & \mu_{24}=144\end{array}$

From these values it is clear than an $A B$ interaction is present between diet history (A) and weeks on diet (B), because frequent dieters do not show as great a change in weight over weeks as infrequent dieters do. Averaging the cell means to obtain the row and column marginal means reveals that main effects of diet history (A) and weeks on diet (B) are also present. To complete the specification, we shall assume that $\sigma=20$ for each cell, and that the correlation between weights across weeks is $\varrho=.99$. This unusually high value is used because individuals' weights relative to each other remain quite stable over time (for the derivation of this value, see Bradley, 1988, p. 54).

Having selected population parameters for our hypothetical study, we are ready to initialize the simulation and generate data. Exhibit 1 illustrates how this is done (note that one or more DATASIM commands, separated by commas, are entered after the "?" prompt, and that the individual tokens of a command are separated by spaces). The top part of Exhibit 1 shows the initialization of the simulation, and the bottom part the simulated data. The first step in initializing the simulation is to specify the research design: DESIGN TWOWAY $2 \times 4 \mathrm{~B} \times \mathrm{S} / \mathrm{A}$ defines the design as a $2 \times 4$ factorial design with repeated measures on factor $B(B \times S)$, and independent groups on factor $A(S / A)$. The number of observations per cell is indicated by NOBS, and the labels for factors A, B, and the dependent variable by the various labeling commands. BVAL indicates the quantitative values for the levels of factor B, and is used for controlling plots of the means. The MU command specifies the population mean $\left(\mu_{\mathrm{ij}}\right)$ for each cell of the design, and SIGMA indicates the standard deviation $(\sigma)$. Finally, BRHO sets the correlation (e) across the levels of factor B (weeks on diet).

Once the simulation is initialized, the DATA command is used to generate and display a simulated data set. It is apparent that the data are highly correlated across columns (factor B), but not across rows (factor A). Exhibit 2 shows several statistical analyses of the simulated data. The sample statistics (STAT) reveal that the simulated data reflect the population trends reasonably well, and the correlation matrix (CORR) shows that cells crossing the levels of $B$ have intercorrelations of about .99 , whereas those crossing the levels of $A$ have intercorrelations of about 0 . The analysis of variance (AOV) shows that the main effect of $B$ and the $A B$ interaction are both significant. These results are consistent with the population and sample trends plotted in Exhibit 3.

Note that the main effect of A was not significant, even though the population means specified for levels $a_{1}$ and 


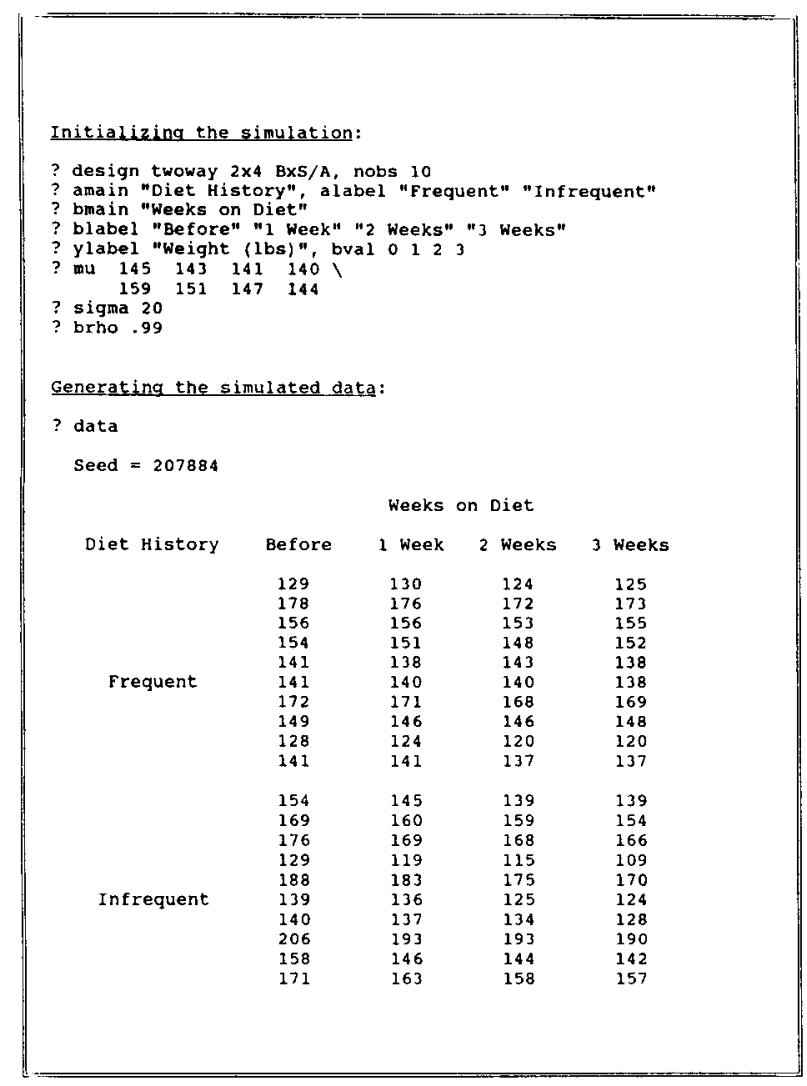

Exhibit 1. Simulating a two-way design with repeated measures on factor $B$.

$\mathrm{a}_{2}$ differ, and the $\eta^{2}$ value for A exceeds that of $\mathrm{B}$. This Type II decision error illustrates an interesting fact about mixed-model experimental designs: namely, that the increased sensitivity of the test conducted on the correlatedgroups factor comes at the expense of the test on the independent-groups factor. This is evident from a comparison of the two error terms: $M S_{\mathrm{s} / \mathrm{a}}=1,679.939$, whereas $M S_{\mathrm{b} \times \mathrm{s} / \mathrm{a}}=3.950$. Given $\mathrm{q}=4$ levels for factor $B$, the expected values of the two error terms are (Winer, 1971, p. 516):

$$
\begin{array}{rlll}
E\left(M S_{\mathrm{s} / \mathrm{a}}\right) & =\sigma^{2}[1+(q-1) \varrho] & =20^{2}[1+(3)(.99)] & =1,588 \\
E\left(M S_{\mathrm{b} \times \mathrm{s} / \mathrm{a}}\right) & =\sigma^{2}(1-\varrho) & =20^{2}(1-.99) & =4
\end{array}
$$

By way of comparison, if a two-way design has independent groups on both factors, then the expected value of the error term for evaluating the main and interaction effects is (Winer, 1971, p. 438):

$$
E\left(M S_{\text {w.cell }}\right)=\sigma^{2}=20^{2}=400
$$

From these formulae we see that $M S_{\mathrm{b} \times \mathrm{s} / \mathrm{a}}<\mathrm{MS}_{\text {w.cell }}<$ $M S_{\mathrm{s} / \mathrm{a}}$ for $\varrho>0$, and that the differences between them increase in proportion to $\varrho$. Therefore, as $\varrho$ goes up in value, the increase in sensitivity of the test on factor $B$ is offset by a decrease in sensitivity of the test on factor A.

The simulated data in Exhibit 1 were generated from normal distributions with $\mu$ and $\sigma$ as defined in the in- itialization. It is possible to have DATASIM generate nonnormally distributed data as well. This is done using the generalized lambda distribution (GLD) developed by Ramberg and Schmeiser (1972, 1974) and Ramberg, Dudewicz, Tadikamalla, and Mykytka (1979):

$$
R(p)=\lambda_{1}+\left[p^{\lambda_{3}}-(1-p)^{\lambda_{4}}\right] / \lambda_{2}
$$

For values of $p$ in the range $0 \leq p \leq 1$, this function defines the value of $X$ [denoted $R(p)$ ] falling at the $p^{\text {th }}$ percentile of the distribution. The lambda parameters $\lambda_{1}$ and $\lambda_{2}$ are location and scale parameters, respectively, and $\lambda_{3}$ and $\lambda_{4}$ are shape parameters that jointly determine the skewness and kurtosis of the distribution. By selecting the right combination of lambda parameters $\left(\lambda_{1}-\lambda_{4}\right)$, one can approximate distributions with varying degrees of skew and tail weight. To obtain a random sample of values, successive calls are made to a uniform random number generator to get values of $p$, and the corresponding values of $X$ calculated from the equation.

The GLD can generate simulated data for highly skewed distributions. We will demonstrate this capability by simulating an actual study, the well-known obedience study by Milgram (1963). To simulate this study, it is necessary to use an exponential distribution, because the majority of Milgram's subjects obeyed all the way to the top of the shock scale (30) and the mode of the distribution was therefore equal to the upper scale limit. The following lambda parameters approximate an ex-

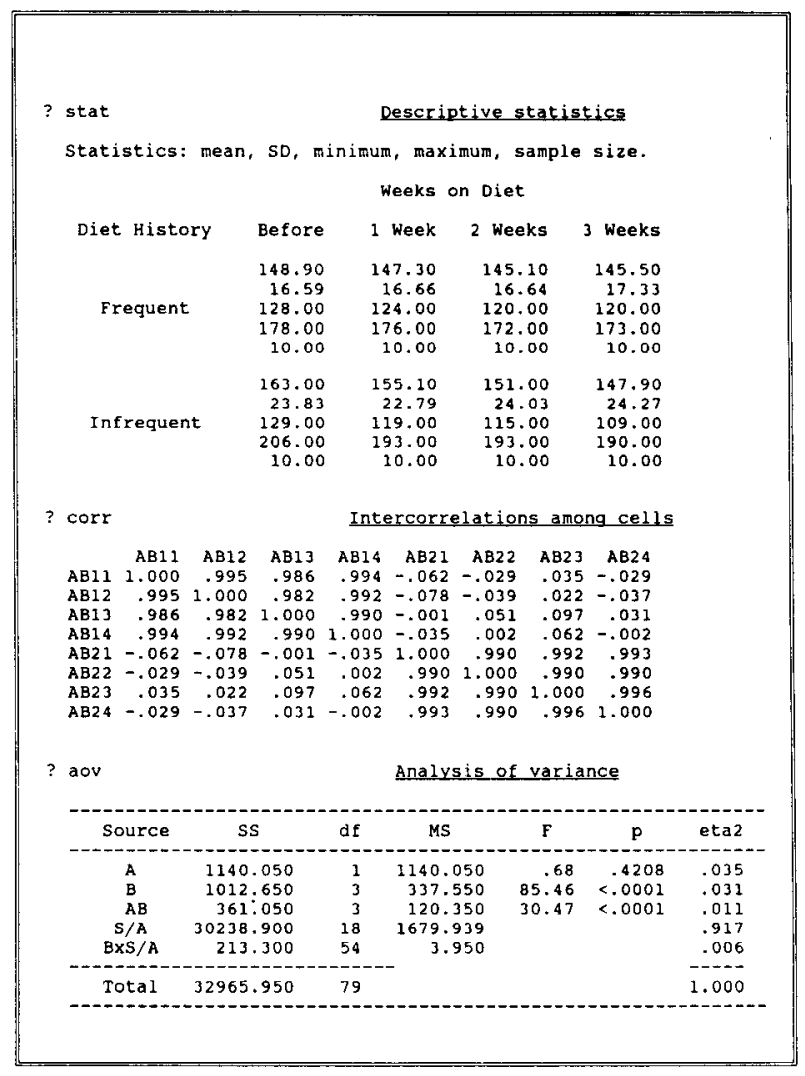

Exhibit 2. Analyzing the simulated data for a $2 \times 4$ design. 


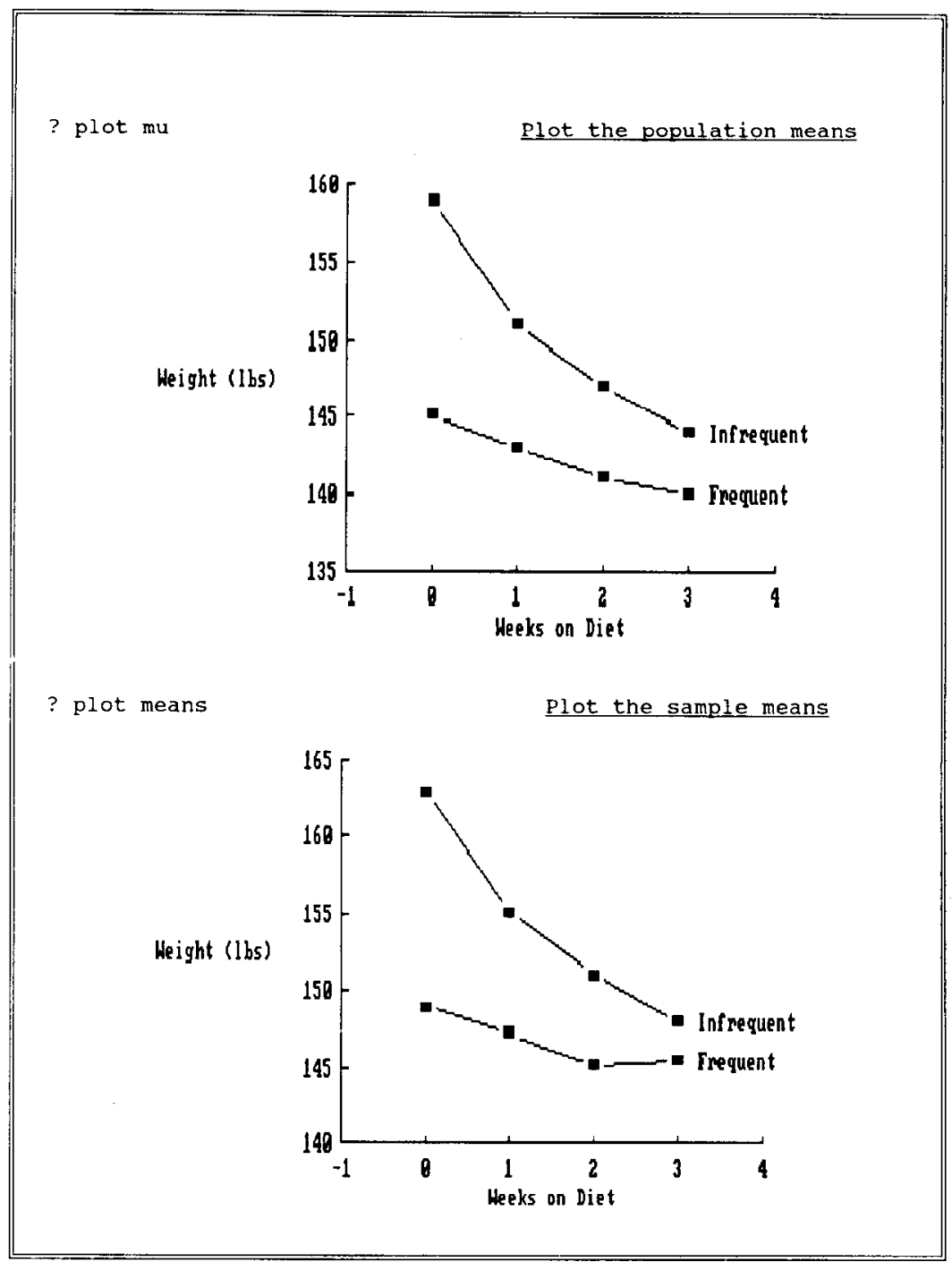

Exhibit 3. Plotting the means for a $2 \times 4$ design.

ponential distribution: $\lambda_{1}=.993, \lambda_{2}=-.001081$, $\lambda_{3}=-.001076$, and $\lambda_{4}=-.00000407$ (Ramberg et al., 1979 , p. 214). These values define a GLD with an extreme degree of negative skew $\left(\alpha_{3}=-2\right)$ and "peakedness" $\left(\alpha_{4}=9\right)$ relative to the normal distribution (see Bradley, 1988, p. 97, for details on how to select lambda parameters for approximating particular nonnormal distributions). To initialize the simulation, we enter:

$$
\begin{aligned}
& \text { DESIGN ONEGROUP, NOBS } 1000 \\
& \text { MU 27.1, SIGMA 3 } \\
& \text { LAMBDA C1 } .993-.001081-.001076-.00000407
\end{aligned}
$$

The design is defined as a one-group design, and the number of observations to be generated is $n=1,000$ (a large sample size is used to provide good resolution for constructing a frequency distribution). The values of $\mu$ and $\sigma$ were estimated from Milgram's $(1963,1965)$ studies, and the lambda parameters were selected so as to generate data with a mode equal to an upper scale limit of 30 . Following this initialization, the DATA command was en- tered to generate a simulated data set of $n=1,000$ values. The STAT command revealed that the sample mean and standard deviation were 26.98 and 2.97 , respectively, with a minimum score of $\mathbf{1 0}$ and a maximum score of 30 . Hence, DATASIM succeeded in generating simulated data with the proper central tendency and variability, while avoiding the off-range values $(X>30)$ that would arise with a normal distribution. We may compare the actual theoretical and empirical distributions by entering the PDF and HIST commands, as shown in Exhibit 4. PDF C1 plots the probability density function of the GLD, and shows that the shock-level data are negatively skewed with a mode of 30 , the highest possible score (the normal distribution superimposed on the PDF indicates the off-range values that would result with this distribution). HIST C1 plots a histogram of the simulated data, and shows that the empirical distribution closely parallels the corresponding PDF.

In summary, this section has demonstrated how to initialize, generate, display, and analyze simulated data for 


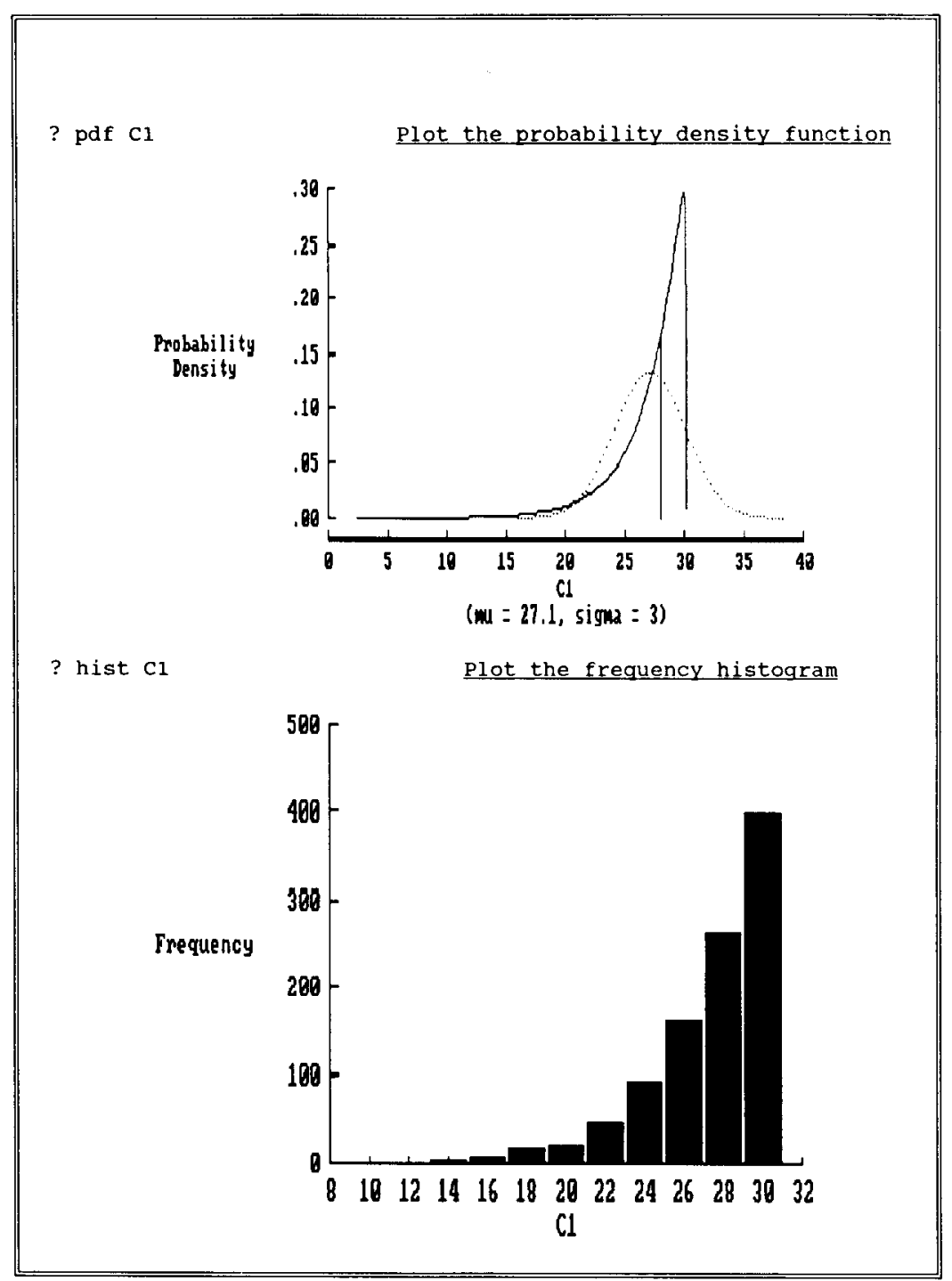

Exhibit 4. Plotting the theoretical and empirical distributions for the simulated data (Milgram, 1963).

hypothetical and actual research studies. While the examples illustrated a $2 \times 4$ factorial design and a one-group design, it is possible to initialize simulations for threeway factorial designs, multivariate designs, one- and twoway contingency-table designs, two-group designs, and the like. In each case, the user specifies a design, sample size, population parameters, and any related information, and then generates a simulated data set using the DATA command. The resulting data may then be analyzed, displayed, and graphed, using the full complement of dataprocessing commands available in DATASIM (Bradley, 1988, chap. 2).

\section{Monte Carlo Investigations}

The use of computers for performing statistical simulations has been an active area of research for over 30 years. Perhaps the most common application involves Monte Carlo simulations for assessing the consequences of violating the assumptions of a statistical test. With this approach, data are randomly sampled from distributions that have characteristics at variance with those assumed by the statistical test (for example, distributions may have nonnormal shapes, heterogeneous variances or covariances, limited scale values, etc.). A statistical test is then applied to the data, and the outcome recorded as significant $(p \leq \alpha)$ or nonsignificant $(p>\alpha)$. A sampling experiment consists of repeating this process a large number of times (typically 1,000 to 10,000 times), and tabulating the number of tests with $p \leq \alpha$. Now, if the distributions are defined in such a way that, however else they might differ, the null hypothesis $\left(H_{0}\right)$ is true, then the proportion of significant tests obtained in the sampling experiment provides an empirical estimate of the probability of making a Type I error. If the consequences of violating assumptions are negligible, then this probability should be similar to the nominal level, $\alpha$. If so, the test is said to be "robust." Conversely, if the distributions are defined such that $H_{0}$ is false, then the propor- 
tion of significant tests obtained in the sampling experiment provides an empirical estimate of the power of the test, $1-\beta$. This value can be compared to the theoretical value calculated with the noncentral distribution for the test statistic being evaluated. If the consequences of violating assumptions are negligible, then the empirical and theoretical values should be similar (when distributions have heterogeneous variances, calculations of "theoretical" power are problematic; see Glass, Peckham, \& Sanders, 1972).

Most psychologists are familiar with the Monte Carlo research of Norton (1952, cited in Lindquist, 1953), who investigated the effects of nonnormality and heterogeneity of variance on the Type I error rate of the $F$ test. By conducting a large number of sampling experiments, Norton demonstrated that the $F$ test is robust to such violations for applications having equal $n_{\mathrm{j}}$ across groups. Similar results were obtained by Hsu and Feldt (1969) and Lunney (1970). Evidence that the $F$ test is robust to heterogeneity of covariance in repeated-measures designs has been provided by Collier, Baker, Mandeville, and Hayes (1967). Glass, Peckham, and Sanders (1972) provide a comprehensive review of the basic strategy and findings of Monte Carlo studies investigating $t$ and $F$.

Monte Carlo simulations have also been conducted to investigate the chi-square statistic. Bradley and Cutcomb (1977) demonstrated that the chi-square test of independence for $2 \times 2$ contingency tables is robust to even severe violations of the minimum-expected-frequency requirement. These findings were subsequently replicated by Camilli and Hopkins (1978). The chi-square test was also found to be robust to violations of minimum expected frequency in larger $(R \times C)$ contingency tables, as reported by Bradley et al. (1979). In these Monte Carlo studies, simulated data are generated by sampling from a discrete probability distribution in which the cell probabilities are equal to the product of the corresponding row and column marginal probabilities: $p_{\mathrm{ij}}=p_{\mathrm{i}} \times p_{\mathrm{j}}$ (given this specification of $p_{\mathrm{ij}}$, the rows and columns are independent, and $H_{0}$ is true). By means of the selection of the proper combination of probabilites and sample sizes, tables having small expected frequencies can be produced. The chisquare test is then conducted on simulated data generated from such probability distributions, and the proportion of significant chi-square tests is compared to the nominal level. Similar procedures have been used by Bradley and Cutcomb (1977) and Bradley and Seely (1977) to assess the consequences of violating the minimum-expectedfrequency requirement on the power of the chi-square test.

The rest of this section will show how DATASIM may be used to replicate and extend the Monte Carlo studies described above. To do this, one simply specifies a research design and population parameters for controlling the simulation. Any number of simulated data sets may be generated and analyzed, the results saved on disk, and the relevant statistics $(F, t$, chi-square, etc.) read into DATASIM and processed accordingly. If one sorts the $p$ levels of the statistical tests, it is a simple matter to de- termine how many tests have $p \leq \alpha$. This number represents the outcome of a single DATASIM sampling experiment.

We can illustrate this process by replicating two of the 147 sampling experiments reported by Bradley and Cutcomb (1977; see their Table 1). As noted above, their research assessed the Type I error rate of the chi-square test in $2 \times 2$ tables having small expected frequencies. At one extreme, they investigated a table with evenly balanced distributions of row and column marginal probabilities: $.5, .5$, and $.5, .5$. At the other extreme, they investigated a table having very unbalanced distributions of row and column marginal probabilities: $.9, .1$, and .9, .1. Under $H_{0}$, the cell probabilities for the balanced table are $.25, .25, .25$, and .25 ; for the unbalanced table they are $.81, .09, .09$, and .01 . When 10,000 samples of $N=4$ observations were generated from each of these probability distributions, the balanced table produced 1,089 significant chi-square tests $(p \leq .05)$, and the unbalanced table 237 . Thus, for tests conducted at a nominal level of $\alpha=.05$, the empirically determined Type I error probabilities are .1089 and .0237 , respectively (by way of comparison, manual expansion of the multinominal results in exact Type I error probabilities of .1094 and .0226). For both tables, then, the actual Type I error probability differs substantially from the nominal level of $\alpha=.05$. However, for samples of $N=10$, Bradley and Cutcomb (1977) found that the error rates for the balanced and unbalanced tables were .0526 and .0481 , respectively. Consequently, for all but the smallest sample sizes, the chi-square test is robust to violations of the minimumexpected-frequency requirement.

With these benchmarks in mind, we can now illustrate using DATASIM to replicate the two sampling experiments just described. Exhibit 5 shows the DATASIM commands for doing this. The first command line specifies the design and sample size for the simulation: DESIGN TABLE $2 \times 2$, NTOT 4 indicates that random samples of $N=4$ observations are to be generated for $2 \times 2$ contingency tables. The next command shown, CPROBS, specifies the cell probabilities for each cell of the table. The values entered correspond to the balanced table described above. At this point, a single simulated data set can be generated and displayed by entering DATA. Note that the seed used to initialize the random number generator is displayed along with the simulated data. This value may be used to regenerate this particular data set at some later time, if desired. Following the data, the CHIS command is entered to conduct a chi-square test on the simulated data set.

The steps illustrated so far are typical of DATASIM simulations: (1) initialize the simulation by specifying a design, sample size, and population parameters; (2) generate a simulated data set; and (3) analyze the data. However, to perform a sampling experiment using Monte Carlo methods, we want to be able to generate a large number of such data sets, and to analyze and store the results of each of them for subsequent analysis. DATA- 


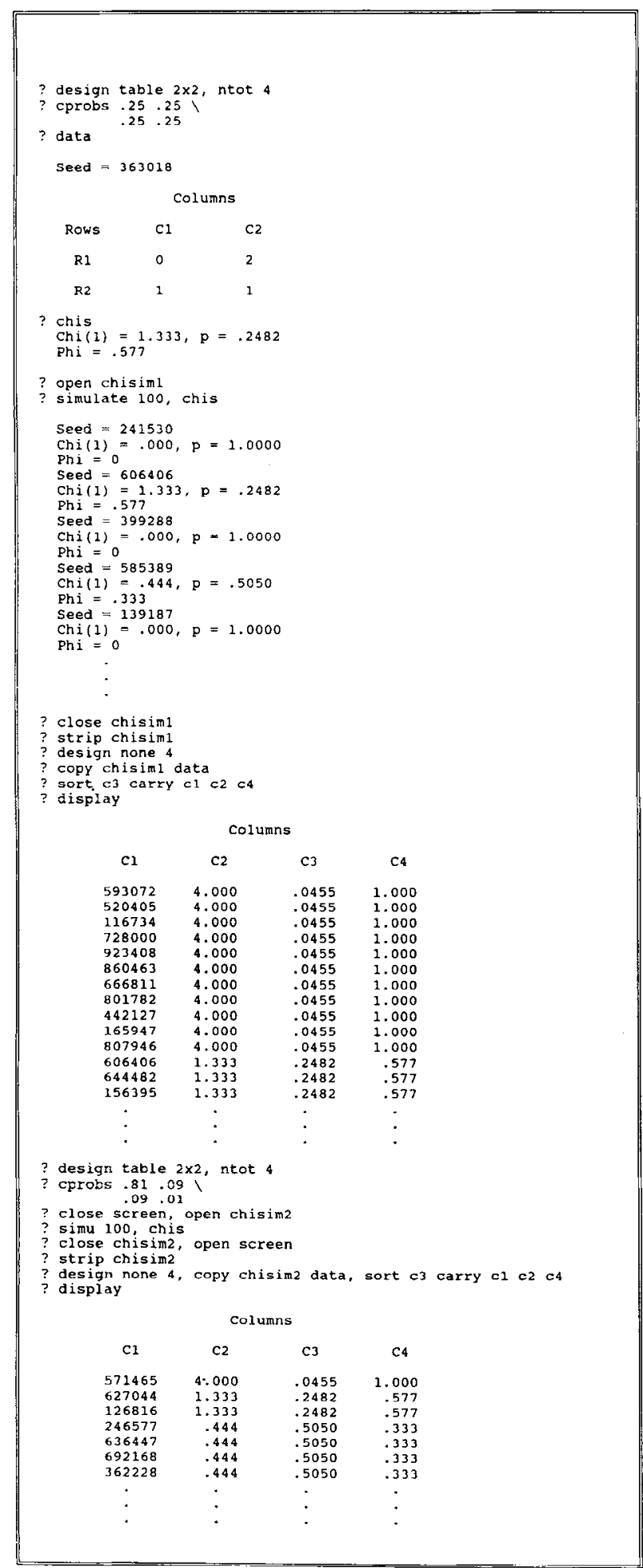

Exhibit 5. Empirical assessment of the Type I error rate of the chi-square statistic in $2 \times 2$ contingency tables.

SIM provides a convenient way to do this. As shown in Exhibit 5, the command OPEN CHISIM1 opens an output file for recording program I/O on disk. With this file open, we now conduct the sampling experiment by entering SIMULATE 100, CHIS. These commands cause
DATASIM to generate 100 simulated data sets and to compute a chi-square test on each. A partial listing of the resulting output is shown in Exhibit 5. For each simulated data set, the seed and the results of the chi-square analysis are displayed. Following the last (100th) simulated data set, the output file is closed by entering CLOSE CHISIM1.

Although the file CHISIM1 stores the results of the sampling experiment, it is not presently in a form that is directly accessible to DATASIM, because the file contains text in addition to the chi-square values and $p$-levels that we are interested in analyzing. The command STRIP CHISIM1 tells DATASIM to process the file by deleting all text, so that only numerical values remain. In addition, all the numerical values associated with a particular seed are combined into a single row of the file. In the present case, this results in the seeds, chi-square values, $p$-levels, and phi coefficients being organized in four columns. Once CHISIM1 has been processed in this way, we can read the numerical values into DATASIM as data. To do this, we first declare a design that can accommodate the four columns of numbers: DESIGN NONE 4 declares the design as "NONE" with 4 columns of data (the design NONE is a catch-all used when the data set to be read into DATASIM does not conform to one of the predefined design types). The command COPY CHISIM1 DATA is then entered to copy the contents of CHISIM1 to the DATASIM data buffer. When the copy operation is complete, the seeds, chi-square values, $p$ levels, and phi values in CHISIM1 will have been copied to columns $\mathrm{Cl}-\mathrm{C} 4$ of the data buffer. Before one displays these numbers, it is convenient to sort them first: SORT C3 CARRY C1 C2 C4 sorts the $p$-levels in C3 and carries along the corresponding values in $\mathrm{Cl}, \mathrm{C} 2$, and $\mathrm{C} 4$. A partial listing of the sorted data is shown in the second part of Exhibit 5, following the DISPLAY command.

The display of the sorted values summarizes the results of the sampling experiment. From the $p$-levels in C3, we see that 11 of the 100 simulated data sets produced chisquare values with $p \leq \alpha$ (because of the discrete nature of the data and the small sample size, the corresponding chi-squares all have values of 4.0 and $p$-levels of .0455). The empirically determined probability of a Type I error is therefore $11 / 100=.11$. This compares to the empirical estimate of $1,089 / 10,000=.1089$ reported by Bradley and Cutcomb (1977), and to the exact value of .1094 computed via the multinomial. This "replication" is not entirely trivial, since the program code and the random number generator used by DATASIM are not the same as those used by Bradley and Cutcomb (1977).

The bottom of Exhibit 5 also shows the DATASIM replication for the table with unbalanced marginals. The commands used to perform the sampling experiment are the same as before, except that different cell probabilities are specified for CPROBS, and the CLOSE SCREEN command is used to suppress the display of simulation I/O on the screen (this speeds things up because the program then only needs to send I/O to the file CHISIM2, which allows the simulation to run in under $2 \mathrm{~min}$ ). Fol- 
lowing the simulation, OPEN SCREEN is used to open the screen again. From the partial listing of the sorted data shown in Exhibit 5, we see that only one of the simulated data sets produced a significant chi-square test $(p=.0455)$. The empirical Type I error probability of $1 / 100=.01$ compares to .0237 obtained by Bradley and Cutcomb (1977), and to .0226 obtained by exact calculation. Since only 100 data sets were generated, the Type I error rates will be $.01, .02, .03$, and so on. Sampling experiments based on larger numbers of data sets $(1,000$ to 10,000 ) provide much better estimates of the actual error rates. Nevertheless, the two sampling experiments shown in Exhibit 5 demonstrate that DATASIM is able to successfully replicate the results of previously published Monte Carlo investigations of the chi-square test.

It is equally easy to conduct DATASIM sampling experiments to investigate the Type I error rate or power of the $t$ or $F$ test. Exhibit 6 illustrates a Monte Carlo simulation investigating the effect of heterogeneity of variance on the Type I error rate of the $F$ test. The commands DESIGN ONEWAY $3 \mathrm{~S} / \mathrm{T}$, NOBS 3 define a one-way experimental design with $k=3$ independent groups and $n=3$ observations per group (the design token $\mathrm{S} / \mathrm{T}$ or $\mathrm{T} \times \mathrm{S}$ is used to indicate if the observations are independent or correlated, respectively). The MU and SIGMA commands define $\mu$ and $\sigma$ for the three populations from which the data will be sampled: $\mu_{1}=\mu_{2}=\mu_{3}=100$, $\sigma_{1}=5, \sigma_{2}=10$, and $\sigma_{3}=15$. Given this specification, $H_{0}$ is true, but the homogeneity-of-variance assumption is false. In fact, the variance of the third population $\left(\sigma_{3}^{2}=225\right)$ is nine times larger than that of the first $\left(\sigma_{1}^{2}=25\right)$. We may now conduct a sampling experiment to determine if this violation substantially alters the Type I error rate of the $F$ test. The SIMULATE command generates 100 simulated data sets, and the AOV 01 command

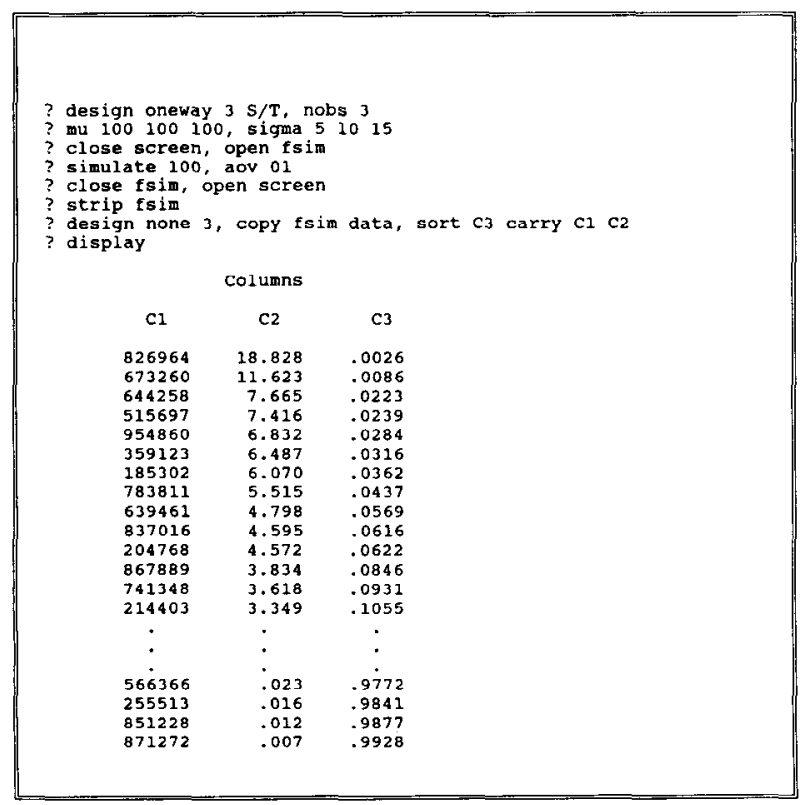

Exhibit 6. Empirical assessment of the Type I error rate of the $F$ test with heterogeneity of variance.

computes the $F$ test for each one. The 01 option for AOV causes just the $F$ ratio and $p$ level to be output, instead of the usual ANOVA summary table. The output for the 100 data sets is stored in the file FSIM and is subsequently stripped of text so that just the seeds, $F$ values, and $p$ levels remain. These three columns of numbers are then read into the three columns of the DATASIM data buffer, and sorted on the basis of the $p$ levels in C3. The sorted values are then displayed with the DISPLAY command. As shown in Exhibit 6, eight of the $F$ tests had $p \leq .05$, so the empirical estimate of the Type I error probability is $8 / 100=.08$.

How well does this sampling experiment correspond to previous Monte Carlo research? Glass et al. (1972; see their Table 8) report the results of a sampling experiment by Norton (1952), which investigated the same combination of parameters as specified in Exhibit 6: $k=3$, $n=3, \sigma_{1}=5, \sigma_{2}=10$, and $\sigma_{3}=15$. Norton's sampling experiment produced an empirical estimate of the Type I error probability of $242 / 3,333=.0726$, as compared to the estimate of .08 obtained by DATASIM. As noted above, greater accuracy may be obtained by increasing the number of simulated data sets: in two additional DATASIM sampling experiments based on 1,000 simulated data sets, empirical estimates of $67 / 1,000=.067$ and $79 / 1,000=.079$ were obtained. The average of these two error rates, .073 , is close to the value reported by Norton (1952).

If DATASIM simulations are initialized such that the population means differ, then sampling experiments may be conducted to assess the power of the $F$ test. Consider the following two initializations:

Initialization 1

DESIGN ONEWAY 3 S/T NOBS 10

MU 100105115

SIGMA 151515
Initialization 2

DESIGN ONEWAY $3 \mathrm{~T} \times \mathrm{S}$
NOBS 10
MU 100105115
SIGMA $15 \quad 1515$
RHO .8

Both initializations define a one-way experimental design with $k=3$ groups, $n=10$ observations per group, and population parameters of $\mu_{1}=100, \mu_{2}=105, \mu_{3}=110$, and $\sigma_{1}=\sigma_{2}=\sigma_{3}=15$. However, the two initializations differ in one important respect: the first defines an independent-groups design ( $\mathrm{S} / \mathrm{T})$, whereas the second defines a correlated-groups design $(T \times S)$. Furthermore, since the second design has correlated observations, the degree of intercorrelation in the population (.8) is specified by RHO. Sampling experiments may be conducted with both of these initializations, and the proportion of significant $F$ tests obtained may be compared to the theoretical values computed using charts (Kepple, 1982, p. 549) or tables (Winer, 1971, p. 886) of the noncentral $F$ distribution.

Following the procedure illustrated in Exhibit 6, 100 simulated data sets were generated and analyzed for each of the two initializations shown above. For Initialization 1, $47 / 100=.47$ of the $F$ tests were significant at $p \leq .05$, 
as compared with $98 / 100=.98$ for Initialization 2 . How do these empirical estimates compare with theory? The value of the noncentrality parameter is (Winer, 1971, p. 221):

$$
\phi=\sqrt{\frac{n \Sigma \tau_{\mathrm{j}}^{2}}{k \sigma_{\mathrm{e}}^{2}}} .
$$

For both initializations, $n=10, k=3$, and $\Sigma \tau_{j}^{2}=$ $\Sigma\left(\mu_{\mathrm{j}}-\mu\right)^{2}=116.67$. For Initialization $1, \sigma_{\mathrm{e}}^{2}=\sigma^{2}=15^{2}$ $=225$, and therefore $\phi=1.315$, with $d f=2,27$. If one interpolates from a table of noncentral $F$ (Winer, 1971, p. 886), one finds that this corresponds to a power of $1-\beta \approx .48$, which is close to the empirical value of .47. For Initialization $2, \sigma_{\mathrm{e}}^{2}=\sigma^{2}(1-\varrho)=225(1-.8)=$ 45 , and therefore $\phi=2.94$, with $d f=2,18$. Interpolating from a table of noncentral $F$, one finds that this corresponds to a power of $1-\beta=.99$, which compares favorably to the empirical estimate of .98 .

As a last example, we illustrate a sampling experiment for assessing the power of a two-sample $t$ test (see Bradley, 1988, p. 96). The following simulation parameters are initialized in DATASIM:

\section{DESIGN TWOGROUP S/T, NOBS 5, MU 50 60, SIGMA 1010}

To perform the sampling experiment, one enters the commands SIMULATE 100 , TWOT C1 C2 to generate 100 simulated data sets, and to conduct a two-sample $t$ test on each. Processing and sorting the results shows that $29 / 100=.29$ of the $t$ tests are significant at $p \leq .05$. Given the initialization, the value of the noncentrality parameter is (Winer, 1971, p. 34):

$$
\begin{aligned}
\delta & =\frac{\mu_{1}-\mu_{2}}{\sigma \sqrt{\left(1 / n_{1}+1 / n_{2}\right)}} \\
& =\frac{50-60}{10 \sqrt{(1 / 5+1 / 5)}}=-1.581 .
\end{aligned}
$$

For a two-tailed $t$ test with $\alpha=.05,8 d f$, and $\delta=-1.581$, the probability of a Type II error is $\beta=.7136$ (this value is obtained using linear interpolation between tabled entries in Winer, 1971, p. 884). The corresponding power of the $t$ test is $1-\beta=.2864$. Hence, the power of the $t$ test estimated by simulation agrees quite well with theory (.29 vs. .2864$)$.

The preceding examples show that DATASIM sampling experiments produce results that agree with theory and with the findings of previous Monte Carlo studies. While we have focused on the chi-square, $F$, and $t$ statistics as applied to data for relatively simple research designs, Monte Carlo simulations can be conducted using any of the statistical analyses performed by DATASIM on the simulated data for any research design. Hence, it is easy to investigate the consequences of violating assumptions on tests involving simple effects, simple-simple effects, simple interactions, linear contrasts, and multiple comparisons in multifactor experimental designs, including designs having a mixture of independent- and correlatedgroups factors. Likewise, it is easy to assess the consequences of violating assumptions on tests involving correlation coefficients, regression coefficients, $R^{2}$, and so on, in multivariate designs. From this perspective, DATASIM is a general purpose tool for conducting Monte Carlo research.

In concluding this section, we should consider the various ways in which simulations may be initialized so as to violate the assumptions of a statistical test. As noted above, DATASIM can initialize simulations for the chisquare test of independence in which the minimumexpected-frequency requirement is violated (for Monte Carlo investigations of the chi-square test of homogeneity, the row or column marginal frequencies may be fixed in value: see Bradley, 1988, p. 61). For $t, F$, and related tests, a variety of assumptions can be violated either singly or in combination. As shown earlier, the homogeneityof-variance assumption is violated by initializing a simulation in which $\sigma$ differs across groups. The normality assumption is violated by initializing nonnormal distributions for one or more groups using the LAMBDA command (this command allows the user to define a large variety of nonnormal symmetric and asymmetric distributions for generating data). The homogeneity-ofcovariance assumption is violated by specifying a correlation matrix in which the values of RHO vary for the different pairs of groups in the design. Finally, for multivariate designs, the LAMBDA command is used to violate the normality assumption made by tests on correlation coefficients, regression coefficients, and $R^{2}$. After such violations have been introduced in the initialization, repetitive simulation may be used to assess the effects of the violations on the Type I error rate or power of a particular statistical test.

\section{DATASIM Homework Assignments}

The previous two sections have illustrated the use of DATASIM to generate simulated data for specific research studies and for Monte Carlo investigations. In both cases, a simulation is initialized by entering appropriate commands for defining the research design, sample size, population parameters, and so forth. In this section, we will see how such initializations can be saved on disk and then used to generate independently randomized data sets for students to analyze and interpret as homework.

Once a simulation has been initialized in DATASIM, the commands used to initialize the simulation may be saved by entering the command SIMSAVE followed by a file name. The simulation may be reinitialized at any time, by entering EXECUTE followed by the file name. Students desiring simulated data would then enter DATA, and an independently randomized data set would be generated and displayed by DATASIM. The students could conduct various analyses on their simulated data sets, and the instructor or an assistant could check their answers by regenerating each student's unique data set and having DATASIM conduct the appropriate analyses. A data set 
is regenerated by entering SEED, followed by the value of the seed assigned to a particular student's data set.

An easier way to use DATASIM in order to generate data for homework assignments is to employ the built-in library of problems supplied with the software. The DATASIM software comes on two diskettes: one contains the executable code for the program, DATASIM.EXE, and the other a library of support files, DATASIM.LIB. The library contains over 40 problems that test the student's ability to analyze and interpret the data for a large variety of research designs. Most DATASIM problems are based on actual research studies published in the literature, many of which would be considered "classic" studies in psychology. Each problem has two files in the library: a description file that contains text describing the research study, and an initialization file that contains DATASIM commands for initializing the simulation. The description and initialization files are stored in the subdirectories DATASIM.LIB $\backslash$ DESCRIPT and DATASIM.LIB $\backslash$ PROBLEMS, respectively. An instructor may add problems to the DATASIM library by simply installing description and initialization files in the appropriate subdirectories of DATASIM.LIB.

The user may obtain brief descriptions of the problems available in the DATASIM library by entering HELP PROB. Exhibit 7 shows a partial listing of the resulting output. Entering DESCRIBE < problem name > produces a complete description of the problem, INITIALIZE < problem name > initializes the simulation, and

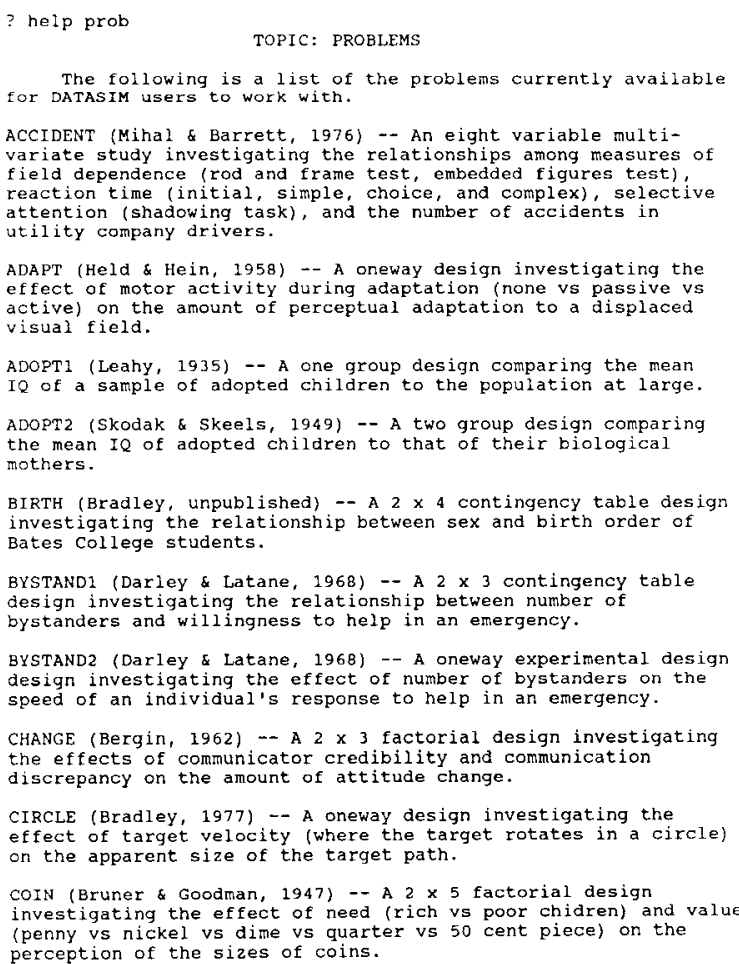

Exhibit 7. Displaying the built-in DATASIM problems.

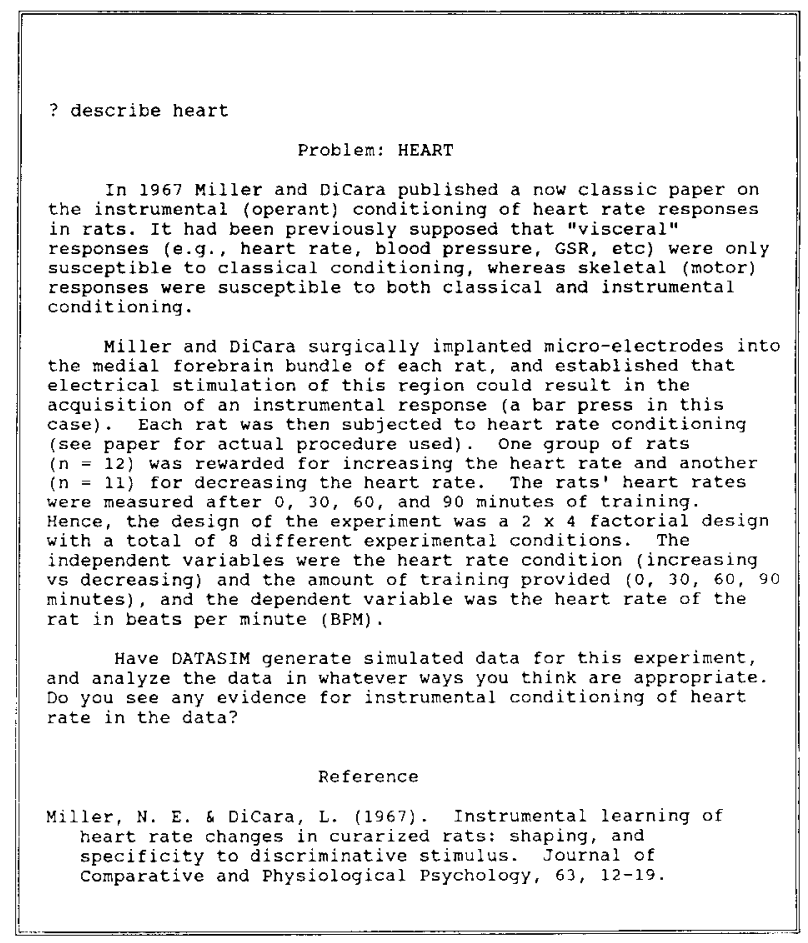

Exhibit 8. On-line description of the problem HEART, a $2 \times 4$ design based on Miller and DiCara (1967).

DATA generates the simulated data. The INITIALIZE command is similar to the EXECUTE command described above, except that it always "looks" for the initialization file in the subdirectory DATASIM.LIB \PROBLEMS. The EXECUTE command looks in the current directory. Another difference is that INITIALIZE does not echo the commands to the screen as they are being executed, as does EXECUTE. This makes the initialization of the simulation "transparent" to the student. As an illustration, consider the commands needed to generate simulated data for the problem ACCIDENT, a multivariate study:

$\begin{array}{ll}\text { OPEN PRINTER } & \text { [open printer for output] } \\ \text { DESCRIBE ACCIDENT } & \text { [describe problem] } \\ \text { INITIALIZE ACCIDENT } & \text { [initialize simulation] } \\ \text { DATA } & \text { [generate simulated data] } \\ \text { CLOSE PRINTER } & \text { [close printer for output] }\end{array}$

The first and last commands simply open and close the printer for output. This allows a hard copy of the problem description and simulated data to be obtained. The other commands describe, initialize, and generate data for the problem.

Exhibits 8-13 illustrate in greater detail the procedure for describing, generating, and analyzing simulated data for a built-in DATASIM problem. The problem, called HEART, is based on the well-known study by Miller and DiCara (1967), which demonstrated that the heart rates of rats could be instrumentally conditioned to increase (the fast group) or decrease (the slow group) in response to ESB reinforcement. Exhibit 8 shows the complete description of this problem. The top of Exhibit 9 shows the initialization file (stored in DATASIM.LIB $\backslash$ PROBLEMS) 


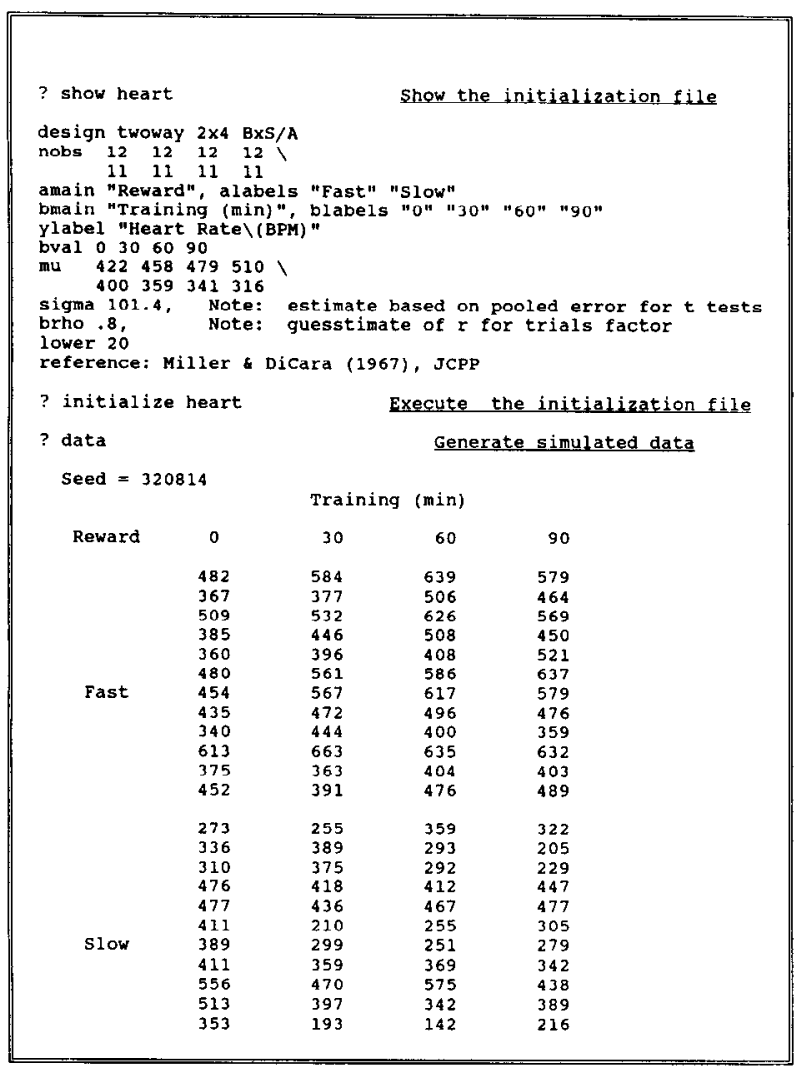

Exhibit 9. Generating a simulated data set for the problem HEART.

displayed by entering SHOW HEART. Students would not normally display the initialization file in the course of doing an assignment; we do so here simply to show the DATASIM commands used to simulate the Miller and DiCara study. As indicated in Exhibit 8, Miller and DiCara (1967) employed a $2 \times 4$ factorial design with independent groups on factor A (fast vs. slow) and repeated measures on factor B (minutes of training). Therefore, the design is specified as DESIGN TWOWAY $2 \times 4$ $B \times$ S/A. Also, since one animal died in the "slow" "reinforcement condition, the sample sizes for the fast $(n=12)$ and slow groups $(n=11)$ were not the same. This is specified by listing the sample size for each cell individually after NOBS. Other commands (AMAIN, ALABEL, etc.) provide labels for factors $A, B$, and the dependent variable. The command BVAL specifies the quantitative values for the levels of factor $B$, which is needed for plots of the means.

The values specified for MU are taken from Figure 1 of Miller and DiCara (1967, p. 14). Unfortunately, the authors did not report standard deviations for the cells, nor did they conduct a two-way ANOVA on the data from which $\sigma$ could be estimated using methods described by Bradley (1988, p. 91). For the same reason, there was no way to estimate the value of $\varrho$ across the levels of factor B. However, Miller and DiCara $(1967$, p. 14) did report the results of two independent $t$ tests: one comparing the heart rates of the fast as opposed to the slow groups prior to training $(0 \mathrm{~min})$, and one comparing their heart rates at the end of training $(90 \mathrm{~min})$. By working backwards, it was possible to obtain an estimate of the withincell variance $\left(s^{2}\right)$ from a knowledge of the $t$ values, means, and sample sizes. The value specified by SIGMA in the initialization file is simply the square root of this estimate. The correlation across the levels of factor B, specified by BRHO, was simply estimated as .8; if a two-way ANOVA had been reported, $\varrho$ would have been computed from the relation $M S_{\mathrm{b} \times \mathrm{s} / \mathrm{a}}=\sigma^{2}(1-\varrho)$.

The bottom of Exhibit 9 shows the initialization of the problem and a simulated data set. INITIALIZE HEART executes the commands in the initialization file (without echoing), and DATA generates and displays the simulated data. Exhibit 10 plots the population (PLOT MU) and sample (PLOT MEANS) cell means for comparison. From these plots, we see that the trends in the simulated data reflect the pattern obtained in the original study. Exhibit 11 presents the results of several statistical analyses performed on the simulated data. The output for the STAT command shows that the sample means and standard deviations are about right, given sampling error, relative to the values of $\mu$ and $\sigma$ specified in the initialization. Likewise, the correlation matrix shows that observations across the levels of the repeated-measures factor (B) are correlated about .8 , as specified (since correlations cannot be computed across cells that differ in sample size, an asterisk is entered in the matrix wherever this is the case).

The bottom of Exhibit 11 shows the results of a mixedmodel ANOVA (AOV) on the simulated data. Consistent with the trends plotted in Exhibit 10, the main effect of factor $A$ and the $A B$ interaction are both significant. Note that since the sample sizes for the two groups (fast vs. slow) are not equal, DATASIM prints a message indicating that an unweighted means analysis has been conducted on the data. As a check on the accuracy of the simulation, we can compare the error mean squares to theoretical expectation (Winer, 1971, p. 516):

$$
\begin{array}{lll}
E\left(M S_{\mathrm{s} / \mathrm{a}}\right)=\sigma^{2}\left[1+(\mathrm{q}-1)_{\varrho}\right] & =101.4^{2}[1+(3)(.8)] & =34,959 \\
E\left(M S_{\mathrm{b} \times \mathrm{s} / \mathrm{a}}\right)=\sigma^{2}(1-\varrho) & =101.4^{2}(1-.8) & =2,056
\end{array}
$$

From the ANOVA output in Exhibit 11, we see that $M S_{\mathrm{s} / \mathrm{a}}=29,176$ and that $M S_{\mathrm{b} \times \mathrm{s} / \mathrm{a}}=2,108$, which are reasonably close to expectation. Pooling these two mean squares to obtain $M S_{\text {w.cell }}$ we have:

$$
\begin{aligned}
& M S_{\mathrm{w} \text {.cell }}=\left(S S_{\mathrm{s} / \mathrm{a}}+S S_{\mathrm{b} \times \mathrm{s} / \mathrm{a})}\right) /\left(d f_{\mathrm{s} / \mathrm{a}}+d f_{\mathrm{b} \times \mathrm{s} / \mathrm{a}}\right) \\
& M S_{\mathrm{w} . \text { cell }}=(612,692.866+132,834.748) /(21+63)=8,875.329
\end{aligned}
$$

The square root of $M S_{\text {w.cell }}, S D_{\text {w.cell }}=94.21$, is reasonably close to the value of $\sigma=101.4$ specified in the initialization. Consequently, the plots in Exhibit 10 and the various statistics in Exhibit 11 indicate that DATASIM is generating simulated data with the desired theoretical properties (central tendency, variability, intercorrelation, mean squares, etc.).

DATASIM can conduct a variety of simple-effect and analytical comparisons for the data from multifactor designs. Since the $A B$ interaction is significant for the 


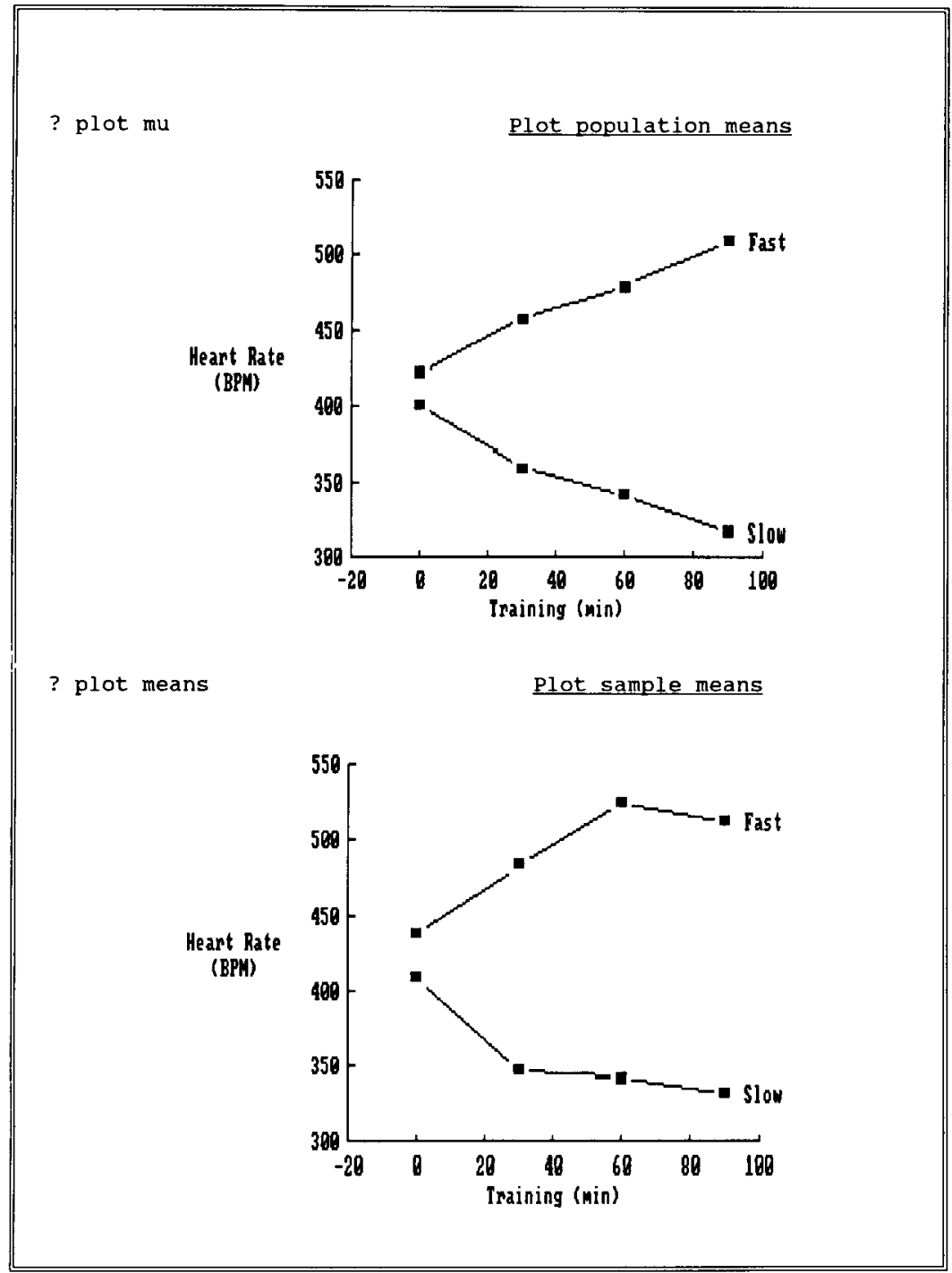

Exhibit 10. Plotting the population and sample means for HEART.

HEART data, it makes sense to conduct simple-effects analyses. Exhibit 12 shows the results of simple-effects tests of $A$ at $b$ and $B$ at $a$. The command SIMPLE A at $b$ compares the mean heart rate of the fast to that of the slow group at each level of training $(0,30,60$, and $90 \mathrm{~min}$ ). Prior to training (i.e., $0 \mathrm{~min}$ ), there is no difference in the heart rates of the two groups $(p=.4799)$. However, for each of the subsequent training periods ( 30 , 60 , and $90 \mathrm{~min}$ ) a significant difference in heart rate is obtained $(p=.0015$ to $p<.0001)$. Miller and DiCara $(1967$, p. 14) reported the following $t$ tests for comparing the mean heart rate of the fast to that of the slow group after $0 \mathrm{~min}$ and after $90 \mathrm{~min}$ of training: $t(21)=.6, \mathrm{NS}$; and $t(21)=4.1, p<.001$, respectively. Converting the corresponding $F$ values ( $A$ at $b_{1}, A$ at $b_{4}$ ) in Exhibit 12 to $t$ values, we have $\sqrt{.51}=.71$ and $\sqrt{21.29}=4.61$. Within the limits of sampling error, these values com- pare favorably to the values reported by Miller and DiCara (1967).

Note that the simple-effects analyses of $A$ at $b$ employ a pooled error term, $M S_{\text {pool }}$, as recommended by Winer (1971, p. 529). In the present case, this pooled error is computed in the same way as was $M S_{\text {w.cell, above. That }}$ is, the two error terms from the overall analysis of variance, $M S_{\mathrm{s} / \mathrm{a}}$ and $M S_{\mathrm{b} \times \mathrm{s} / \mathrm{a}}$, are pooled as follows:

$$
M S_{\text {pool }}=\left(S S_{\mathrm{s} / \mathrm{a}}+S S_{\mathrm{b} \times \mathrm{s} / \mathrm{a}}\right) /\left(d f_{\mathrm{s} / \mathrm{a}}+d f_{\mathrm{b} \times \mathrm{s} / \mathrm{a}}\right)=8,875.329
$$

The pooled $d f$ for this error term is $d f_{\text {pool }}=84$, as noted in the output for the simple-effects tests in Exhibit 12. However, because $M S_{\text {pool }}$ involves pooling heterogeneous sources of variation, for purposes of testing it cannot be considered to have $d f_{\text {pool }}=84$ degrees of freedom. Winer $(1971$, p. 530$)$ recommends an adjusted $d f$ based on the Satterthwaite approximation, which results in 


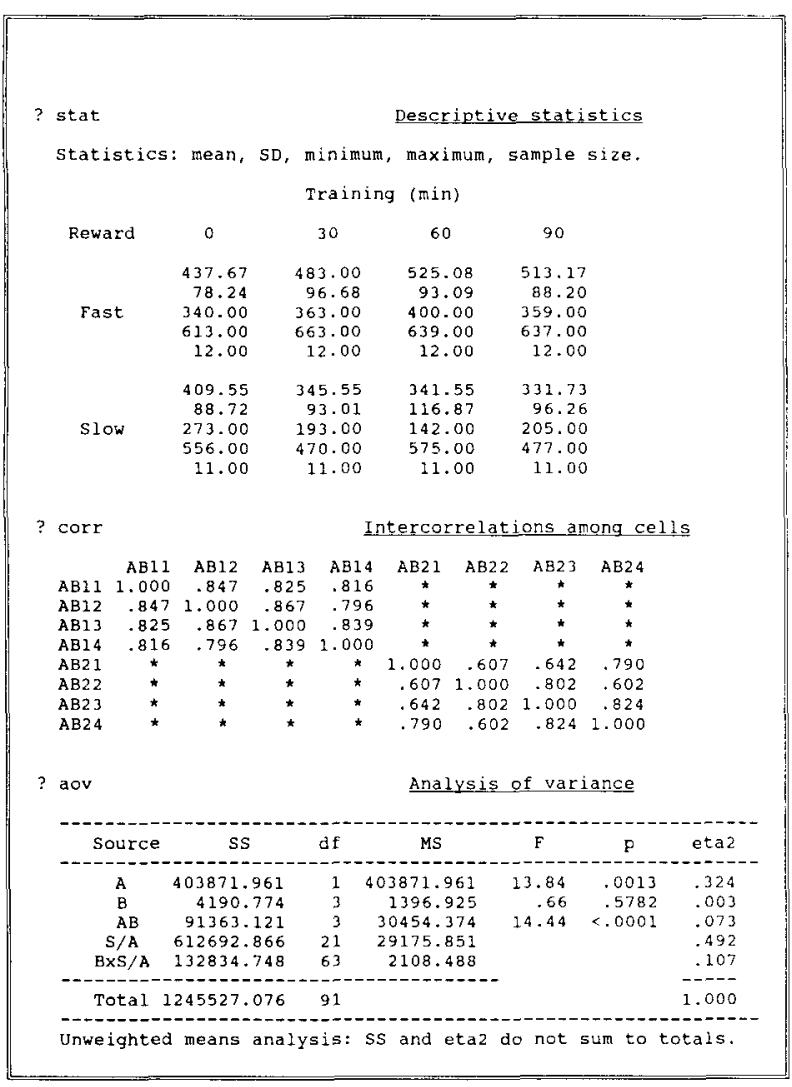

Exhibit 11. Analyzing the simulated data for the problem HEART.

$d f_{\text {adj }}=31$. DATASIM uses this adjusted $d f$ for computing the $p$-levels of any $F$ tests employing the $M S_{\text {pool }}$ error term (this is not an issue for the simple-effects analyses of $B$ at $a$, because these $F$ tests employ $M S_{\mathrm{b} \times \mathrm{s} / \mathrm{a}}$ rather than a pooled error term).

In addition to simple-effects tests, we may conduct a number of additional analytical comparisons. Exhibit 13 shows multiple pairwise comparisons, linear contrasts, and trend analysis of the simulated data for HEART. The command COMPARE B at al performs multiple pairwise comparisons of the cell means in the first row $\left(a_{1}\right)$ of the data matrix; that is, of the heart rates of the fast group after $0,30,60$, and $90 \mathrm{~min}$ of training. The command COMPARE B at a 2 does the same for the slow group (both sets of results are consistent with the trends plotted for the sample means in Exhibit 10). The command CONTRAST B at a2 3-1-1-1 performs a linear contrast of the means in the second row $\left(a_{2}\right)$ of the data matrix; that is, it compares the heart rate of the slow group after $\mathbf{0}$ min of training to the heart rate of the slow group averaged across 30,60 , and 90 min of training. Finally, the command CONTRAST B at al $-1+1+1-1$ performs a linear contrast of the means in the first row of the data matrix. Since the contrast coefficients $(-1+1+1-1)$ correspond to those for testing a quadratic trend component, this actually performs a test of trend on the data of the fast group. In this case, the quadratic component is significant, which is consistent with the trend shown in Exhibit 10 for the sample data. Since Miller and DiCara's (1967) data showed little, if any, quadratic trend, this result might be regarded as a Type I error. Any of the analytical comparisons illustrated in Exhibit 13 can be conducted at the main effect level as well, assuming this makes sense.

The problem HEART represents just one of many DATASIM problems based on factorial designs. In addi-

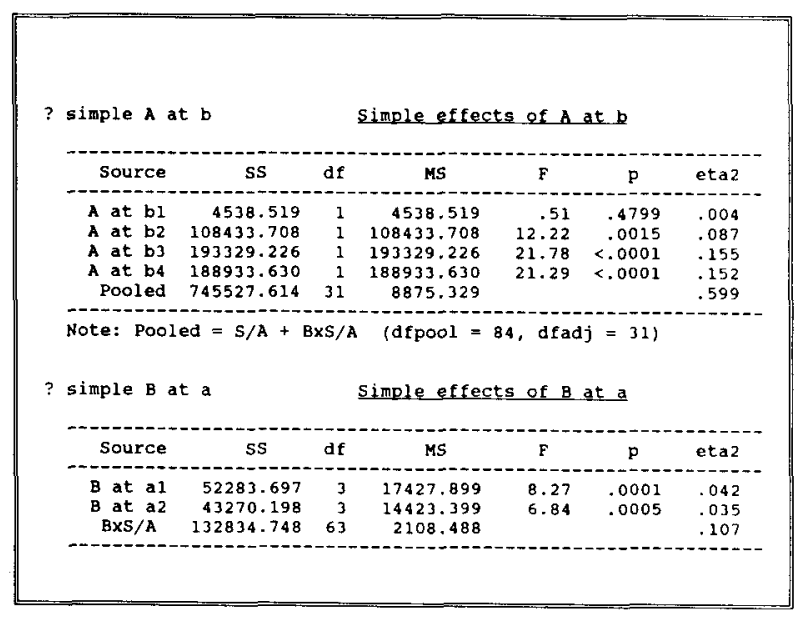

Exhibit 12. Simple-effects analyses of the data for HEART.

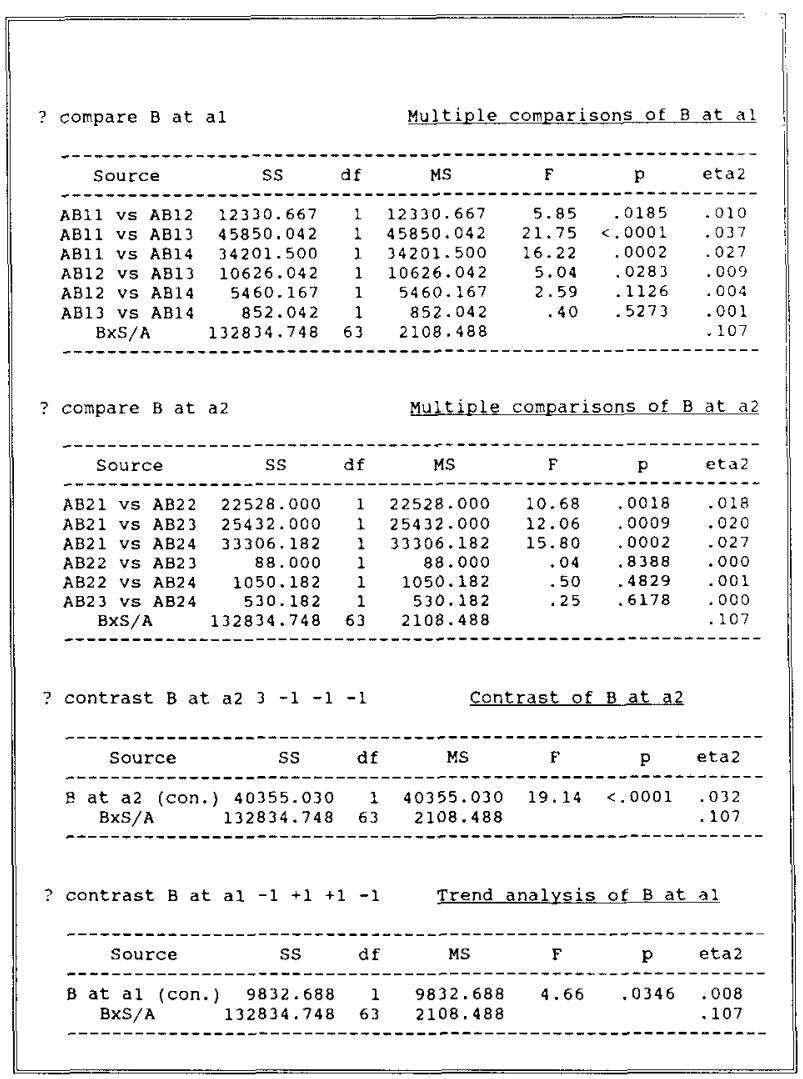

Exhibit 13. Analytical comparisons of the data for HEART. 
tion, problems are available for one-group, two-group, one-way, correlational, multivariate, and table designs. Some of these are described briefly in Exhibit 7. When assigned a given problem, students simply use the DESCRIBE, INITIALIZE, and DATA commands to obtain the description and simulated data. DATASIM can also be used to analyze the data, or to verify that the solutions obtained from some other source (hand calculation, another software package, etc.) are correct. The built-in problems are also useful for CAI demonstrations in the classroom, because simulated data for a variety of research designs can be easily displayed, analyzed, and graphed on-line.

\section{Summary}

DATASIM is a general-purpose data simulator for simulating research studies, conducting sampling experiments and Monte Carlo investigations, generating and analyzing data for homework assignments, and performing simulations that illustrate various principles of sampling theory. Data sets can be generated with normal or nonnormal distributions, independent or correlated observations, and equal or unequal sample sizes. For table designs, the row and/or column marginal totals can be allowed to vary randomly, or to be fixed by the sampling scheme. The DATASIM software provides a built-in library of problems with description and initialization files that instructors and students can use in courses in statistics, experimental design, and research methods. These various capabilities make the program very useful for CAI demonstrations in the classroom. Finally, most users will find the DATASIM data-analysis routines, especially the ANOVA and analytical-test routines, convenient for analyzing their own raw data.

\section{REFERENCES}

BradiEy, D. R. (1978). An interactive data-generating and answercorrecting system for problems in statistics. Behavior Research Methods \& Instrumentation, 10, 218-227.

BRADLEY, D. R. (1984a). Computer simulation of classic studies in psychology. Proceedings \& Abstracts of the Eastern Psychological Association, 55, 60.

BraDLEY, D. R. (1984b). DATASIM: A general purpose simulation program. Proceedings of the Practicum in Computing in Undergraduate Psychology at Gettysburg College, 11-18.

BradLey, D. R. (1988). DATASIM. Lewiston, Maine: Desktop Press.

BRADLEY, D. R. (in press). A general purpose simulation program for statistics and research methods. In S. Nagel \& G. Garson (Eds.), $A d-$ vances in social science and computers (Vol. 1). Greenwich, CT: JAI Press.
Bradley, D. R., Bradley, T. D., McGrath, S. G, Curcome, S. D. (1979). Type I error rate of the chi-square lest of independence in $\mathrm{R} \times \mathrm{C}$ tables that have small expected frequencies. Psychological Bulletin, 86, 1290-1297.

Bradley, D. R., Cutcomb, S. (1977). Monte Carlo simulations and the chi-square test of independence. Behavior Research Methods \& Instrumentation, 9, 193-201.

Bradley, D. R., Hotchkiss, C. M., Dumais, S. T., \& Shea, S. L. (1976). Computer assisted instruction in the small college. Proceedings of the Seventh Annual Conference on Computing in the Undergraduate Curricula, 205-213.

Bradley, D. R., \& SEely, D. L. (1977). Empirical determination of the power of the chi-square test of independence in $2 \times 2$ tables. Proceedings of the Statistical Computing Section of the American Statistical Association, 138-144.

CAMLLI, G., HopXINs, K. D. (1978). Applicability of the chi-square to $2 \times 2$ contingency tables with small expected frequencies. Psychological Bulletin, 85, 163-167.

Collier, R. O., Baker, F. B., Mandeville, G. K., \&ayes, T. F. (1967). Estimates of test size for several test procedures based on conventional variance ratios in the repeated measures design. Psychometrika, 32, 339-353.

Glass, G. V., Peckham, P. D., \& Sanders, J. R. (1972). Consequences of failure to meet assumptions underlying the fixed effects analyses of variance and covariance. Review of Educational Research, 42, 237-288.

Hsu, T., \& FeLdT, L. S. (1969). The effect of limitations on the number of criterion score values on the significance level of the F-test. American Educational Research Journal, 6, 515-527.

KEPPEL, G. (1982). Design and analysis: A researcher's handbook (2nd ed.). Englewood Cliffs, NJ: Prentice-Hall.

LINDQUisT, E. F. (1953). Design and analysis of experiments in education and psychology. Boston: Houghton Mifflin.

LUNNEY, G. H. (1970). Using analysis of variance with a dichotomous dependent variable: An empirical study. Journal of Educational Measurement, 7, 263-269.

Milgram, S. (1963). Behavioral study of obedience. Journal of $A b$ normal \& Social Psychology, 67, 371-378.

Milgram, S. (1965). Some conditions of obedience and disobedience to authority. Human Relations, 18, 57-76.

Miller, N. E., \& DiCARA, L. (1967). Instrumental learning of heart rate changes in curarized rats: Shaping, and specificity to discriminative stimulus. Journal of Comparative \& Physiological Psychology, 63, 12-19.

NokTon, D. W. (1952). An empirical investigation of some effects of non-normality and heterogeneity of the F-distribution. Unpublished doctoral dissertation, Iowa State University, Ames.

Ramberg, J. S., Dudewicz, E. J., Tadikamalla, P. R., MykytKa, E. F. (1979). A probability distribution and its uses in fitting data. Technometrics, 21, 201-214.

RAMHERG, J. S., \& SchMEISER, B. W. (1972). An approximate method for generating symmetric random variables. Communications of the $A C M, 15,987-990$.

RAMBeRG, J. S., \& SCHMEISER, B. W. (1974). An approximate method for generating asymmetric random variables. Communications of the $A C M, 17,78-82$.

WINER, B. J. (1971). Statistical principles of experimental design (2nd ed.). New York: McGraw-Hill. 